\title{
Constructing as-is BIMs from progressive scan data
}

\author{
Te Gao ${ }^{1}$, Burcu Akinci ${ }^{1}$, Semiha Ergan ${ }^{1}$, James H. Garrett, Jr ${ }^{1}$ \\ ${ }^{1}$ Department of Civil and Environmental Engineering, Carnegie Mellon University, \\ Pittsburgh, PA, USA \\ *Corresponding author (tgao@andrew.cmu.edu)
}

\begin{abstract}
Purpose Building Information Models (BIMs) have the potential to support various engineering applications (e.g., building energy analysis, renovation and retrofit planning, and facility management) in the facility operation phase. It is important to keep the information stored in as-is BIMs, accurate and up-to-date. Laser-scanning technology is able to capture the as-is geometric condition of a facility in a timely manner. Hence, the laser scan data can be used as the reference to construct an as-is BIM. However, due to the occlusions caused by furniture, machinery, and building components, a single laser scan might only capture a partial view of a facility, which limits the value of laser scan data in the construction of as-is BIMs. Method In order to overcome this limitation, we propose to perform multiple laser scans of a facility during the construction, renovation or retrofit processes, and fuse the laser scan data captured at different times to create as-is BIMs. The purpose of this paper is to develop a formal approach to evaluate and compare the progressive laser scan data, and identify the value of using progressive laser scan data to create an accurate (i.e., the as-is BIM represents the actual as-is geometric condition of the facility) and complete (i.e., the as-is BIM contains all the components required to be modeled for the facility) as-is BIM. Results \& Discussion We conducted a case study to present the process of creating an as-is BIM from progressive laser scan data, and identified the tasks that could be automated. We selected a research lab that was recently renovated as the testbed. In the case study, we performed multiple laser scans during the renovation process of the research lab to capture the geometric information of the lab at different phases of the renovation process. Figure 1 shows examples of the progressive laser scan data of the research lab. We formally assessed the progressive laser scan data in terms of their geometric accuracy and the represented components. The results showed that the progressive laser scan data can be used to eliminate static occlusions introduced during the construction, renovation or retrofit processes, and can be used to generate a complete view of the facility that covers all visible (e.g., walls, ceilings, floors) and invisible (e.g., airducts, water pipes that are hidden behind the finished surfaces) components with accurate geometries.
\end{abstract}

Keywords: information technology, progressive laser scanning, as-is BIMs

\section{INTRODUCTION}

Used as the semantically rich representations of facilities, Building Information Models (BIMs) are capable of providing the as-is condition of facilities to support various operation and maintenance activities. However, in order to utilize BIMs in the facility operation phase, it is important to keep information stored in BIMs accurate and complete, since the documented facility information quickly becomes outdated due to changes happening in a facility over time ${ }^{2}$.

While it is possible to construct as-is BIMs directly from design drawings, many buildings do not have up-to-date design drawings available. The renovation, maintenance and repair activities might change the configurations of buildings, and these changes are not recorded in a consistent way ${ }^{3}$. Another way to construct as-is BIMs is to update the as-designed model developed in the design phase of a facility into an as-is model. However, since a facility does not always get constructed exactly as the design specifies, extensive surveying is needed to measure the differences between the as-designed model and the as-built conditions ${ }^{4}$. As an alternative to these approaches, laser scanning technology has been wide- ly used in the industry to capture the detailed as-is conditions of facilities. Laser scanning technology has the capability to efficiently capture the 3D geometry of a facility in the form of point clouds. In current practice, point cloud data is used as a reference to manually generate as-is BIMs with modeling tools (e.g., Revit, ArchiCAD). The general modeling process is composed of three steps: (a) manually identifying the building elements to be modeled from the point cloud data (e.g., finding the points belonging to a pipe that needs to be modeled); (b) tracing the points to determine the location and the dimension of the building elements; and (c) modeling the elements with the modeling tools. This general modeling process is limited by the occlusions that existed when the point cloud data was collected. Due to the occlusions caused by temporary construction materials/equipment, furniture and building elements, the elements to be modeled might not be visible or only be partially visible in the point cloud data captured at a single point in time. For example, HVAC air ducts, which are installed above the ceiling tiles in a facility, would not be visible in the laser scans performed after the installation of the ceiling tiles. 


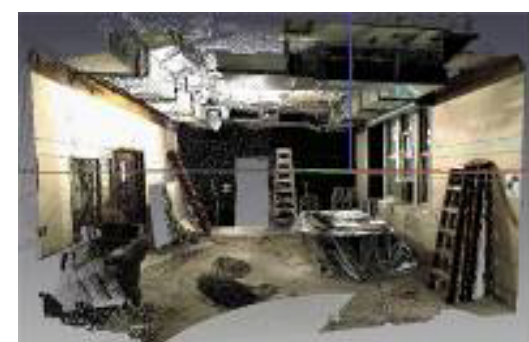

Figure 1a. Laser scan data captured in May

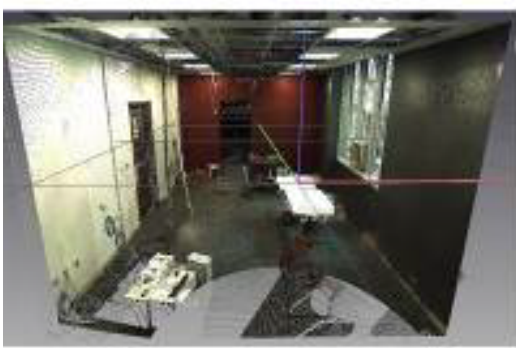

Figure 1c. Laser scan data captured in July

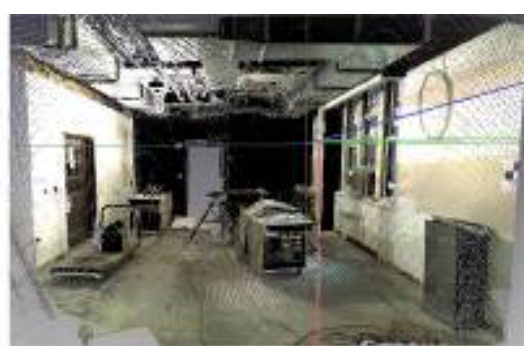

Figure 1b. Laser scan data captured in June

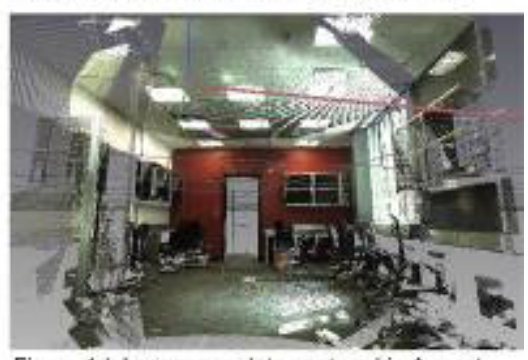

Figure 1d. Laser scan data captured in August

Fig. 1. The progressive laser scan data of the research lab

Research studies have been performed concerning the creation of BIMs from point cloud data. Tang et al. (2010) proposed an automated process to convert point cloud data into a BIM. This process is divided into three steps: (a) fitting geometric primitives to point cloud data; (b) recognizing objects from point cloud data; and (c) establishing the relationships among different elements ${ }^{4}$. Currently there is no state-of-the-art method that can be solely used to accomplish all the three steps of the automated process to convert point cloud data into as-is BIMs ${ }^{3}$. A wide variety of methods have been proposed to recognize and extract planar surfaces from the point cloud data ${ }^{5,6}$. There is a sizeable amount of work done in the computer vision domain to identify and extract 3D objects from the point cloud data based on the features, such as spin-images and shape descriptors ${ }^{4,7,8}$. Following these methods, the identified planar surfaces or $3 \mathrm{D}$ objects can be used to form the elements in as-is BIMs. However, the occlusions existing in a scene can challenge the results obtained from these approaches. When a facility is progressively scanned over time, the likelihood of having the same occlusions at exactly the same parts of the building components is likely going to be reduced and hence would likely result in a more complete depiction of the scene.

In order to capture a complete view of a facility, we propose to scan the facility at different times while the facility is under construction or major renovation/retrofit. The point cloud data captured progressively over time is referred to as progressive laser scan data in this paper. To evaluate the benefits of using progressive laser scan data to create as-is BIMs, we used a research lab that was recently renovated as the testbed and progressively scanned the lab at different phases of the renovation process. The progressive laser scan data has been evaluated in two aspects: (a) the accuracy of the geometric information provided by the progressive laser scan data; and (b) the completeness of the geometric information that the progressive laser scan data is able to provide in order to model the required building elements in as-is BIMs. In this paper, we mainly focus on the completeness analysis of the progressive laser scan data, since the accuracy analysis cannot be performed until the objects are identified and recognized from the point cloud data. We assessed the completeness of the progressive laser scan in two steps: (a) identifying the building elements in the point cloud data that were captured at a certain time; and (b) evaluating whether building elements are fully visible, partially visible or invisible in the progressive laser scan data. The hypothesis here is that with the progressive laser scan data, one would expect to obtain a more complete view of building elements than using the point cloud data captured at a single point in time. The overview of the case study and its associated research findings are discussed in details in the following sections.

\section{Case Study overview}

A case study was conducted on a four month renovation project in a one hundred year old university campus building. In this project, three adjacent rooms were renovated to accommodate a high-tech research lab. The scope of this renovation project included the installation of high-end analytics equipment, 3D immersive visualization equipment and a new HVAC system. As the renovation was progressing, the research team visited the renovated space on several occasions and performed multiple laser scans to capture the interior of the research lab at different phases of the renovation process. At each point in time, the research team performed multiple scans from different locations to cover all the interior surfaces of the lab. Figure 2 shows a portion of the 
progressive laser scan data captured in the research lab from May 2011 to August 2011.

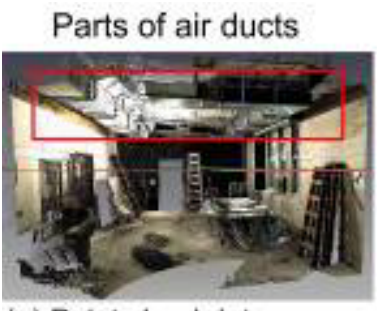

(a) Point cloud data

captured in May

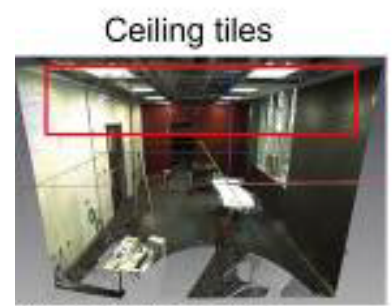

(c) Point cloud data captured in July

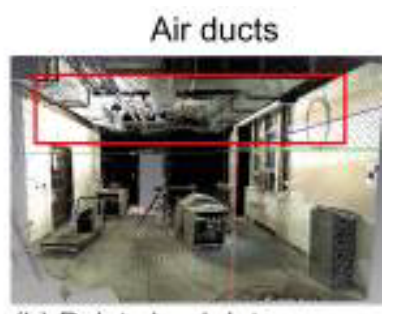

(b) Point cloud data captured in June

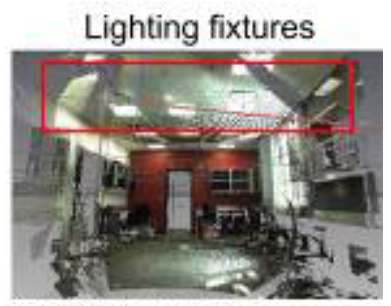

(d) Point cloud data captured in Aug
Fig. 2. The progressive laser scan data of the research lab, highlighting the building elements captured at the ceiling of the room

As seen in Figure 2, the point cloud data captured at different times contain different geometric information for different building elements. With the renovation in progress, additional building elements were installed in the lab and are shown in the corresponding point cloud data. Those installed elements might obscure the vision of the laser scanner, and thus caused various occlusions in the point cloud data. For example, the air ducts were installed above the ceiling of the research lab. The point cloud data captured in May only contains parts of the air ducts since not all of the ducts were installed at that time. The point cloud data captured in June shows all the air ducts, since they were all installed by that time. However, the air ducts are invisible in the point cloud data captured in July and August because of the occlusions caused by the newly installed ceiling tiles.

In addition to the building elements which might be invisible in the progressive laser scan data, the building elements that are visible in all the progressive laser scans might also lack sufficient geometric information for modeling. For example, as seen in Figure 2, the walls, windows and doors are visible in all the point cloud data captured between May to August. Modeling of these elements would require certain attributes (e.g., dimensions, locations) to be extracted from the point cloud data. However, due to the occlusions or noise in the point cloud data, some of the required attributes are invisible or partially occluded in some of the scans. In both cases (i.e., visible and invisible elements), the modelers might not have all the required geometric information (e.g., shape, location, dimensions) from the laser scan performed at a single point in time. The problem highlights the opportunity of combining the point cloud data captured at different times in order to remove the occlusions that can result in visible building elements with partially missing geometric information or invisible building elements. A detailed assessment of the progressive laser scan data is presented in the next section.

\section{ASSESSMENT OF THE PROGRESSIVE LASER SCAN DATA}

In this section, we describe the work we have done to understand the completeness of the information required to model building elements from progressive laser scans. The assessment has been performed from two perspectives. The first part of the assessment is called the visibility analysis at object level and included understanding whether the building elements to be modeled are visible, partially visible or invisible in a scene, and labeling them as such. The second part of the assessment is called the visibility analysis at attribute level, which incorporated analysis of attributes required to model a given building element and performed only for the building elements that were labeled as partially visible in the object level analysis.

\section{Visibility analysis of the progressive laser scans at object level}

We reviewed the progressive laser scan data and identified three labels to classify the building elements captured in a given laser scan:

(a) Fully visible: A building element in a given point cloud data is completely visible for all the attributes required to model that building element. One example of this category is shown in Figure 3(a). In this example, the wall is labeled as fully visible since all dimensions (length, width and height) as well as its location are fully seen in the point cloud data without any occlusions.

(b) Partially visible: A building element in a given point cloud data is partly occluded and at least one of the attributes required to model that building element is missing in that scan. As seen in Figure 3(b), the right part of the wall is missing in the point cloud data, which limits a modeler to see the boundaries of the wall as well as to get the length of the wall. Hence the wall shown in Figure 3(a) is labeled as a partially visible element.

(c) Invisible: A building element cannot be seen in a given point cloud data. When a building element is labeled as invisible, it might be either not installed at the time when the laser scan was performed or fully occluded by other objects (e.g., construction material/equipment and surrounding building elements). 


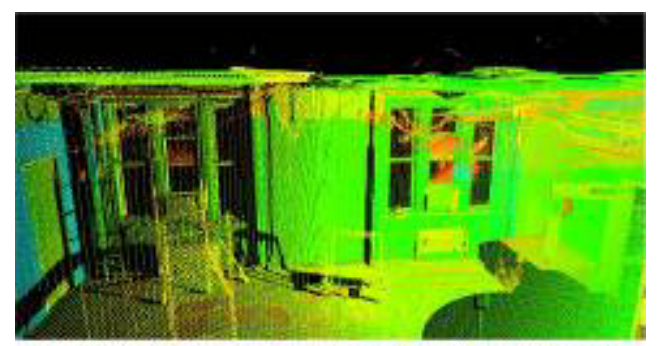

(a) Fully visible: the wall is completely visible in the point cloud data.

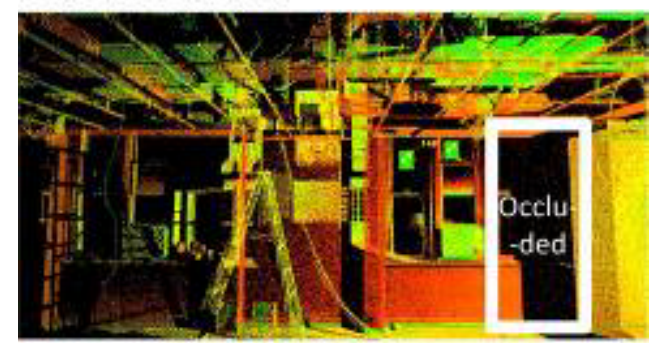

(b) Partially visible: the right part of the wall is occluded.

Fig. 3. An example laser scan showing two walls labeled as fully (a) and partially (b) visible

We analyzed the building elements in each of the progressive laser scans in terms of their visibility and categorized them using the labeling scheme introduced above. In this paper, we report the work in relation to the architectural elements, i.e., doors, windows, lighting fixtures, ceilings and walls. The floor plan of the research lab is shown in Figure 4, where the architectural elements are shown with their IDs. These IDs is used to label their visibilities in each scan.

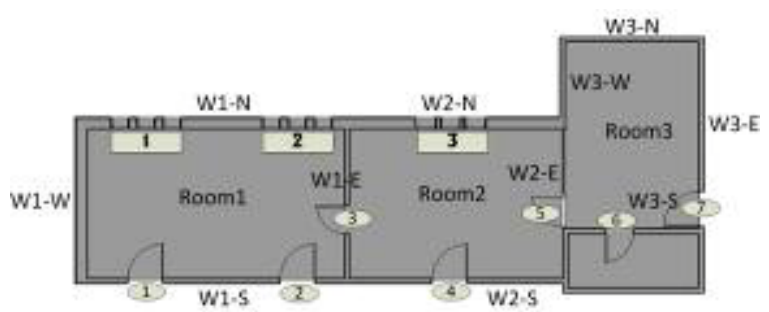

Fig. 4. The floor plan of the research lab with the building elements shown with their IDs

The results of this analysis are shown in Figure 5. From May to August, the research team scanned the site at six different times. At each time, the research team scanned the site from different locations and registered the point clouds from each scan together into the same coordinate system. Hence, each laser scan shown in Figure 5 is referring to the registered point cloud data that was captured at a single point in time.

\begin{tabular}{|c|c|c|c|c|c|c|c|}
\hline Symbols: & \multicolumn{2}{|c|}{$\begin{array}{l}\text { Fully } \\
\text { Visible }\end{array}$} & \multicolumn{2}{|c|}{$\begin{array}{l}\text { Partially } \\
\text { Visible }\end{array}$} & D & Invisible & 0 \\
\hline \multicolumn{8}{|c|}{ Figure 4(a). Building elements in Room 1} \\
\hline $\begin{array}{l}\text { Laser } \\
\text { Scan }\end{array}$ & $\begin{array}{l}21- \\
\text { May }\end{array}$ & $\begin{array}{l}24- \\
\text { May }\end{array}$ & $\begin{array}{l}28- \\
\text { May }\end{array}$ & $\begin{array}{l}\text { 21- } \\
\text { Jun }\end{array}$ & \begin{tabular}{|l|}
$12-$ \\
Jul
\end{tabular} & $\begin{array}{l}\text { 21- } \\
\text { Jul }\end{array}$ & $\begin{array}{l}\text { 14- } \\
\text { Aug }\end{array}$ \\
\hline W1-W & ? & $\mathbf{C}$ & $\mathbf{0}$ & C & $\mathbf{C}$ & $\mathbf{C}$ & C \\
\hline W1-N & $\mathbf{0}$ & $\mathbf{C}$ & $\mathbf{0}$ & C & $\mathbf{0}$ & C & $\mathbf{C}$ \\
\hline W1-E & ? & ○ & ? & O & ? & $\mathbf{C}$ & $\mathbf{C}$ \\
\hline W1-s & - & - & - & - & $\mathbf{0}$ & $\mathbf{C}$ & $\mathbf{C}$ \\
\hline Window1 & $\mathbf{0}$ & $\mathbf{0}$ & (1) & (1) & $\mathbf{C}$ & $\mathbf{C}$ & $\mathbf{C}$ \\
\hline Window2 & $\mathbf{C}$ & $\mathbf{C}$ & $\mathbf{C}$ & $\mathbf{C}$ & $\mathbf{C}$ & ○ & ? \\
\hline Door1 & P & ? & P & O & O & ○ & P \\
\hline Door2 & ? & - & ? & ○ & ○ & ○ & P \\
\hline Door3 & - & ○ & ○ & - & ○ & - & - \\
\hline $\begin{array}{c}\text { Raw } \\
\text { Ceiling }\end{array}$ & - & - & - & - & $\mathbf{0}$ & C & $\mathrm{O}$ \\
\hline $\begin{array}{l}\text { Ceiling } \\
\text { Tiles }\end{array}$ & 0 & $\mathrm{O}$ & 0 & $\mathrm{O}$ & ○ & ○ & P \\
\hline $\begin{array}{l}\text { Lighting } \\
\text { Fixture }\end{array}$ & 0 & $\mathrm{O}$ & 0 & $\mathrm{O}$ & ○ & - & - \\
\hline \multicolumn{8}{|c|}{ Figure 4(b). Building elements in Room 2} \\
\hline $\begin{array}{l}\text { Laser } \\
\text { Scan }\end{array}$ & $\begin{array}{l}21- \\
\text { May }\end{array}$ & $\begin{array}{l}24- \\
\text { May }\end{array}$ & $\begin{array}{l}28- \\
\text { May }\end{array}$ & $\begin{array}{l}21 \text { - } \\
\text { Jun }\end{array}$ & $\begin{array}{l}12 . \\
\text { Jul }\end{array}$ & $\begin{array}{l}21- \\
\text { Jul }\end{array}$ & $\begin{array}{l}14- \\
\text { Aug }\end{array}$ \\
\hline W2-N & 0 & 0 & 0 & 0 & 0 & 0 & 0 \\
\hline W2-E & ○ & ○ & ○ & ○ & ○ & C & C \\
\hline W2-S & 0 & 0 & O & - & ○ & C & C \\
\hline Window3 & - & ○ & - & ○ & - & - & - \\
\hline Door4 & - & - & - & - & 0 & ○ & C \\
\hline Door5 & 0 & 0 & 0 & 0 & 0 & 0 & - \\
\hline $\begin{array}{l}\text { Raw } \\
\text { Ceiling }\end{array}$ & - & - & - & - & C & 0 & 0 \\
\hline $\begin{array}{l}\text { Ceiling } \\
\text { Tiles }\end{array}$ & 0 & 0 & 0 & 0 & - & - & - \\
\hline $\begin{array}{l}\text { Lighting } \\
\text { Fixture }\end{array}$ & 0 & 0 & 0 & 0 & 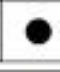 & - & \\
\hline
\end{tabular}

\begin{tabular}{|c|c|c|c|c|c|c|c|}
\hline \multicolumn{8}{|c|}{ Figure 4(c). Building elements in Room 3} \\
\hline $\begin{array}{l}\text { Laser } \\
\text { Scan }\end{array}$ & $\begin{array}{l}21 . \\
\text { May }\end{array}$ & $\begin{array}{l}24 . \\
\text { May }\end{array}$ & $\begin{array}{l}28- \\
\text { May }\end{array}$ & $\begin{array}{l}\text { 21- } \\
\text { Jun }\end{array}$ & $\begin{array}{l}\text { 12. } \\
\text { Jul }\end{array}$ & $\begin{array}{l}\text { 21- } \\
\text { Jul }\end{array}$ & $\begin{array}{l}14- \\
\text { Aug }\end{array}$ \\
\hline w3-w & - & - & 0 & - & - & - & C \\
\hline W3-N & - & - & - & - & - & - & C \\
\hline W3-E & - & - & - & • & • & - & 0 \\
\hline W3-s & - & - & • & - & D & - & C \\
\hline Door6 & - & - & - & - & - & - & - \\
\hline Door7 & - & - & - & • & - & - & 0 \\
\hline $\begin{array}{l}\text { Raw } \\
\text { Ceiling }\end{array}$ & - & - & - & - & C & C & 0 \\
\hline $\begin{array}{l}\text { Ceiling } \\
\text { Tiles }\end{array}$ & 0 & 0 & 0 & 0 & - & $\bullet$ & - \\
\hline $\begin{array}{l}\text { Lighting } \\
\text { Fixture }\end{array}$ & 0 & 0 & 0 & 0 & 0 & 0 & - \\
\hline
\end{tabular}

Fig. 5. Visibility analysis of building elements at object level

As shown in Figure 5, among the seven laser scans performed at different phases of the renovation process, none of them solely is capable of providing all of the architectural elements as fully visible. Out of the eleven walls in the lab, wall W1-N is partially visible in all of the seven laser scans. In order to get a full view of the rest of the walls, more than one laser scan must be performed at different times. Out of the five doors in the lab, three of them are fully 
visible in all of the seven laser scans. Door 5 is visible only in the point cloud data captured on August 14, because it was not installed until August. Due to the occlusions caused by the surrounding building elements and temporary equipment, there is no laser scan that is capable of presenting window 1 as fully visible. The ceiling tiles and lighting fixtures are invisible in the laser scans performed before July, whereas the laser scans performed in July and August successfully capture the geometric surfaces of ceiling tiles and light fixtures, but fail to fully capture the raw ceilings (i.e., raw ceilings are labeled as invisible or partially visible in these laser scans). The reason behind it is that the ceiling tiles and lighting fixtures were not installed until July, and after they were installed, they occluded the raw ceilings, which made the raw ceilings invisible or partially visible. The major benefit of progressive laser scanning is seen in such cases where multiple scans can be utilized to get a complete view of the elements that are partially captured in different scans to model them with as-is dimensions. Therefore, in order to model different building elements in as-is BIMs, we need to refer to the point cloud data captured at different times and select the point cloud data that is able to provide the most complete view of the building elements.

\section{Visibility analysis of the progressive laser scans at attribute level}

As seen in Figure 5, some of the building elements, such as wall $\mathrm{W} 1-\mathrm{N}$ and window 1 , are partially occluded in all of the progressive laser scans. Since there is no point cloud data that is capable of providing a full view of such elements, an alternative is to retrieve the geometric information from the laser scans performed at different times, and combined the geometric information together to model the building elements that are partially visible. Figure 6 gives an example of how point cloud data captured at different times can be combined together to provide the full visibility of a wall to help the modeling activities. In Figure 6(a), the right side of the wall is not presented in the point cloud data captured on June 21. Whereas in Figure 6(b), the point cloud data captured on August 14 shows the right side of the wall, but fails to show the details of the center part of the wall. If we combine the point cloud data captured at these points in time, it is possible to get a full visibility of the wall, and retrieve all the attributes (e.g., location dimensions, orientation, and shape) required to model the wall.

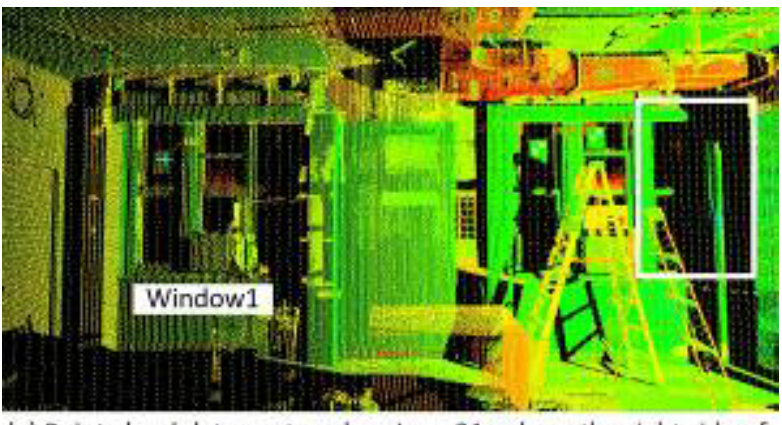

(a) Point cloud data captured on June 21, where the right side of the wall is partially occluded

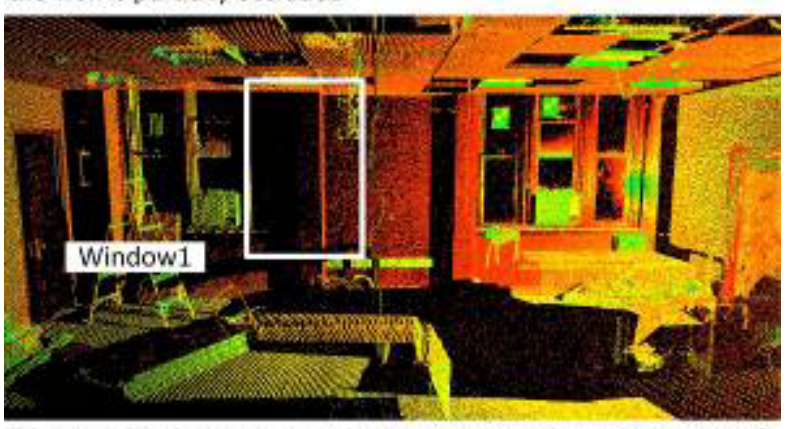

(b) Point cloud data captured on August 14, where the center of the wall is partially occluded

Fig. 6. An example of Wall W1-N and Window1 shown in the point cloud data captured at two different points in time

According to the visibility analysis of the progressive laser scans at object level, the north wall of room 1 (W1-N) is partially occluded in all of the progressive laser scans. Therefore, in this section we looked at wall $\mathrm{W} 1-\mathrm{N}$ in more details. We first identified the attributes that are required to model the geometric representation of wall $\mathrm{W} 1-\mathrm{N}$ in the as-is BIM, and then evaluated each of the progressive laser scans in terms of the visibilities of these attributes in the scan data.

In order to identify the attributes for modeling wall W1-N, we referred to Industry Foundation Classes (IFC), which is an open data model standard to represent and exchange building information ${ }^{9}$. In the IFC schema, a wall can be presented by the IfcWallStandardCase class. The 3D shape of a wall in the IfcWallStandardCase class could be modeled by the sweep representation approach, where the $3 D$ shape of the wall is constructed by sweeping the $2 \mathrm{D}$ surface (i.e., the bottom of the wall) along a certain direction ${ }^{10}$. As seen in Figure 7, in order to model the wall in the IfcWallStandardCase class, the following attributes are required ${ }^{9}$ : (a) Xdim, i.e., the length of the wall; (b) Ydim, i.e., the thickness of the wall; (c) ExtrudedDirection, i.e., the direction of the extrusion; (d) Position, i.e., the position of the wall, which is usually defined by the $3 D$ coordinate of the center of the swept area, and (e) Depth, i.e., the height of the wall. 


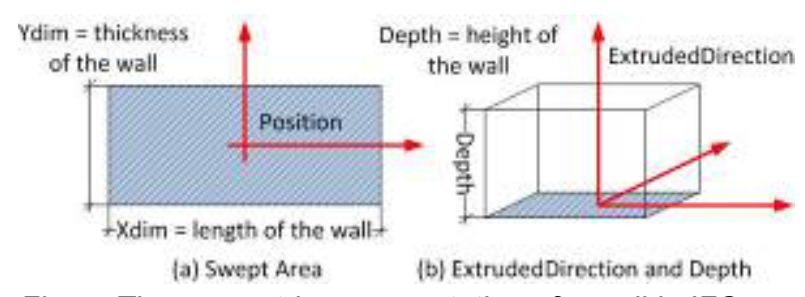

Fig. 7. The geometric representation of a wall in IFC schema

We used the same labeling scheme (fully visible, partially visible and invisible) to investigate the attributes of wall W1-N presented in the progressive laser scan data. The results of the analysis are shown in Figure 8. Among the seven laser scans performed at different phases of the renovation process, only one scan captures the length of the wall as fully visible. In the other laser scans, the length of the wall cannot be fully retrieved, since the edges of the wall are occluded. The position of the wall can be derived from the point cloud data by finding the interaction of wall W1-N with its adjacent walls. The height of the wall is partially visible in the laser scans in July and August, since the ceiling tiles that were installed in July occluded the top part of the wall. In most of the cases, the extruded direction is vertical, and perpendicular to the floor ground. Since we can get the floor plan from the progressive laser scans, the extruded direction is visible in all of the progressive laser scans. However, the thickness of the wall is missing in all of the progressive laser scans. The reason is that laser scans were performed at the interior of the research lab, and the exterior surface of the lab was not captured. Hence, one way to get the wall thickness is to scan the exterior surface of the lab, and then register the interior and exterior scans together. Another way is to fuse the point cloud data with other data sources, such as design drawings or product submittals, to get all the required attributes.

According to the analysis, different point cloud data might contain different geometric information of the partially visible elements. Although a building element is partially occluded in all of the progressive laser scans, it is still possible to retrieve all the required attributes from different scans and combine them to model the geometric representation of building elements. For instance, the laser scan performed on July 21 provides the attributes, i.e., the length of the wall, the extruded direction, and the position, for modeling wall $\mathrm{W} 1-\mathrm{N}$, whereas the laser scans performed in May or June fully capture the height of the wall. Although the thickness of the wall cannot be gained from the progressive laser scans, we can refer to the other data sources, such as design drawings, or specifications to get this attribute. Therefore, combining the progressive laser scans and the other data sources (e.g., specifications, design drawings), we could gather all the required attributes to model the wall in an as-is BIM.

\begin{tabular}{|c|c|c|c|c|c|c|c|}
\hline \multirow[b]{2}{*}{ Attributes } & \multicolumn{7}{|c|}{ Progressive Laser Scan Data } \\
\hline & $\begin{array}{l}21- \\
\text { May }\end{array}$ & $\begin{array}{l}24- \\
\text { May }\end{array}$ & $\begin{array}{l}\text { 28- } \\
\text { May }\end{array}$ & $\begin{array}{l}21- \\
\text { Jun }\end{array}$ & $\begin{array}{l}\text { 12- } \\
\text { Jul }\end{array}$ & $\begin{array}{l}21- \\
\text { Jul }\end{array}$ & $\begin{array}{r}\text { 14- } \\
\text { Aug }\end{array}$ \\
\hline $\begin{array}{c}\text { Xdim } \\
\text { (length) }\end{array}$ & O & D & D & O & D & O & D \\
\hline $\begin{array}{c}\text { Ydim } \\
\text { (thickness) }\end{array}$ & $\mathrm{O}$ & $\mathrm{O}$ & $\mathrm{O}$ & $\mathrm{O}$ & $\mathrm{O}$ & $\mathrm{O}$ & $\mathrm{O}$ \\
\hline $\begin{array}{l}\text { Depth } \\
\text { (Height) }\end{array}$ & 0 & 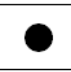 & 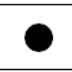 & & O & D & D \\
\hline $\begin{array}{l}\text { Extruded } \\
\text { Direction }\end{array}$ & & & & & & & \\
\hline Position & C & 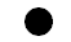 & 0 & 0 & 0 & ? & \\
\hline
\end{tabular}

Fig. 8. Geometric information provided by the progressive laser scan data for a wall

\section{CONCLUSIONS AND FUTURE WORK}

Point cloud data captured by laser scanners can be used as the reference to create as-is BIMs. However, the changing facility environment and the dynamic construction or renovation process introduce occlusions in the point cloud data and obscure the elements that need to be modeled. This problem highlights the opportunity of using progressive laser scans to create as-is BIMs. We conducted a case study to assess the completeness of the point cloud data captured at different times and identify the value of using progressive laser scan data to support the construction of as-is BIMs. The results indicated that the progressive laser scan data can be used to eliminate occlusions introduced during the construction, renovation or retrofit processes, and can be used to generate a complete view of the facility that covers all visible (e.g., wall, ceiling, floor) and invisible (e.g., air ducts and water pipes that are hidden behind finished surfaces) elements.

The future work of this research is to develop a formal approach to configure the progressive laser scans and use it to help the construction of as-is BIMs. To develop such an approach, the first step is to develop the reasoning mechanisms to assess the quality of the progressive laser scan data in terms of accuracy and completeness and configure the scans captured at different points in time in order to get all the required attributes for the modeling activities. In addition, point cloud data only provides geometric information, whereas an as-is BIM needs a variety of other facility information, such as material information, construction cost, warranty information and so on. Hence, in order to construct a semantically rich as-is BIM, information from other data sources (e.g., specifications, project cost database, product submittal) need to be extracted and fused into the as-is BIM. In the second step, we will identify the data sources that are capable of providing the required facility information for the as-is BIM, and de- 
velop a framework to encode the knowledge and information coming from different data sources (e.g., specifications, point cloud data, design drawings) into the process of creating a complete and accurate as-is BIM.

\section{References}

1. Tang, P., Anil, E.B., Akinci, B., and Huber, D., "Efficient and Effective Quality Assessment of As-is Building Information Models and 3D Laser-scanned Data", ASCE Workshop of Computing in Civil Engineering, 2011.

2. Akcamete, A., Akinci, B., and Garrett Jr., J.H., "Towards A Formal Approach for Updating Building Information Models", $5^{\text {th }}$ International Conference on Innovation in Architecture, Engineering and Construction, Antalya, Turkey, 2008.

3. Goedert, J., and Meadati, P. "Integrating Construction Process Documentation into Building Information Modeling", Journal of Construction Engineering and Management, Vol. 134, pp. 509, 2008.

4. Tang, P., Huber, D., Akinci, B., Lipman, R., and Lytle, A. "Automatic Reconstruction of As-built Building Information Models from Laser-scanned Point Clouds: A Review of Related Techniques", Automation in construction, Vol. 19(7), pp. 829-843, 2010.

5. Rabbani, T., Heuvel, F.A. van den, and Vosselman, G., "Segmentation of Point Clouds using Smoothness Constraint", International Archives of Photogrammetry, Remote Sensing and Spatial Information Sciences, Vol. 36(5), pp. 248-253, 2006.

6. Xiong, X. and Huber, D. "Using Context to Create Semantic 3d Models of Indoor Environments", Proceedings of the British Machine Vision Conference, pp. 45.1-45.11. BMVA Press, September 2010.

7. Adan, A., Xiong, X., Akinci, B., and Huber, D. "Automatic Creation of Semantically Rich 3D Building Models from Laser Scanner Data", Proceedings of the International Symposium on Automation and Robotics in Construction (ISARC), June, 2011.

8. Huber, D., Kapuria, A., and Donamukkala, R. "Parts-based 3D Object Classification", Proceedings of the IEEE Conference on Computer Vision and Pattern Recognition, (CVPR), pp. 82-89, 2004.

9. "IFC2x Edition 3 Technical Corrigendum 1", http://buildingsmarttech.org/ifc/IFC2×3/TC1/html/index.htm, retrieved on April 1, 2012.

10. Eastman, C.M., "Building Product Models: Computer Environments Supporting Design and Construction", CRC, 1999. 